\title{
Limitations in the use of Barrier Buckets in a Beta Beam Decay Ring
}

\author{
C. Hansen ${ }^{\dagger, 1}$, E. Wildner ${ }^{\dagger}$, E. Fernandez Martinez $z^{\ddagger}$ \\ ${ }^{\dagger}$ CERN, Geneva, SWITZERLAND \\ ${ }^{\ddagger}$ Max-Planck-Institut für Physik, München, GERMANY
}

\begin{abstract}
Beta Beam, the concept of letting accelerated radioactive ions generate a pure and intense (anti-) neutrino beam by beta decay, is the base of one of the proposed next generation neutrino oscillation facilities, necessary for a complete study of the neutrino oscillation parameter space. The first CERN based scenario, using ${ }^{18} \mathrm{Ne}$ and ${ }^{6} \mathrm{He}$ as $\nu_{e}$ and $\bar{\nu}_{e}$ emitters respectively, has shown a shortfall in ${ }^{18} \mathrm{Ne}$ production. We have now entered the era of the studies of an alternative CERN based baseline. This alternative investigates how the ions ${ }^{8} \mathrm{~B}$ and ${ }^{8} \mathrm{Li}$ can be produced in high quantities in a "Production Ring", accelerated and then accumulated in a "Decay Ring". The idea of an alternative RF scheme for the Decay Ring, to use RF barriers to collect all ions in one "Barrier Bucket", has been tested. Longitudinal phase space simulations show that with the Barrier Bucket method it is unfeasible to optimize between ion intensity in the bucket and the duty cycle the bucket occupy. Therefore the prior RF scheme, that proved to accomplish a duty cycle that followed the requirements, will have to be investigated also for the new ions.
\end{abstract}

\section{Introduction}

Within the EURISOL Design Study (part of FP6 [1]) a CERN based Beta Beam scenario wherein the ions ${ }^{18} \mathrm{Ne}$ and ${ }^{6} \mathrm{He}$ were proposed to be accelerated to $\gamma=100$ and the emitted neutrinos $\left(\nu_{e}\right.$ and $\bar{\nu}_{e}$ respectively) to be detected in a $440 \mathrm{kt}$ Čerenkov detector in the Fréjus tunnel at $L_{F P 6}=130 \mathrm{~km}$ from CERN. For injection into the Decay Ring (DR) a novel method was developed to keep the duty cycle (DC) low [2]. The suppression factor (SF) of the detector, which is the same value as the DC of the DR, is thereby also low which is needed to keep the atmospheric neutrino background rate low. This method gave 5.2 ns short bunches. Twenty of those add up to $\mathrm{DC}=\mathrm{SF}=0.45 \%$ ( $\mathrm{SF}<1 \%$ required for FP6 [3]).

Within EUROnu (part of FP7 [4]) it is being studied the possibility to use ${ }^{8} \mathrm{~B}$ and ${ }^{8} \mathrm{Li}$, both at $\gamma=100$, as $\nu_{e}$ and $\bar{\nu}_{e}$ source respectively. Due to higher Q values the neutrino energies will be higher so that the oscillation maximum will be further away from CERN and therefore the Čerenkov detector will be located at a longer baseline at around $L_{F P 7}=700 \mathrm{~km}$. As a preliminary approach for the FP7 scenario we will use the FP6 scenario as a base structure. For the ion production the result of the production ring suggested by [5] will be assumed to work for both ${ }^{8} \mathrm{~B}$ and ${ }^{8} \mathrm{Li}$, i.e. $10^{14}$ ions/s. Here we will study the possibility to use an alternative RF method for the DR. The idea is to use voltage barriers to squeeze all incoming ions from SPS into one so called Barrier Bucket. High intensity of ions inside the bucket increases the neutrino flux and thereby also the sensitivities of the experiment. The time spread of the bucket would however decrease the sensitivities since that would worsen the SF of the experiment. We have studied the possibility to optimize between the ion intensity kept inside the bucket and the duty cycle that the bucket occupy so that the sensitivities comply with the requirements for the Beta Beam.

\section{Barrier Buckets}

To collect ions in a Barrier Bucket, voltage barriers are needed. Very wide-band cavities, designed for the most common type of barriers, single RF sinusoid, would not manage to deliver as high voltage barriers as needed for our operations. Therefore our investigations were based on the use of the same type of traveling wave (TW) cavities as those that have already performed high voltage Barrier Bucket

\footnotetext{
${ }^{1}$ Corresponding author: Christian.Hansen@cern.ch
} 
operations in the SPS [6]. For the simulations we assumed three barriers, each with a frequency of $200 \mathrm{MHz}$ and a maximum voltage of $8 \mathrm{MV}$. The filling time, equal to the ratio of the length of the TW cavity and the group velocity; $\tau=L_{T W} / v_{g}$, is the crucial barrier parameter. For SPS $\tau=600 \mathrm{~ns}$ [6]. Longitudinal phase space simulations, [7] and [8], showed that for the DR shorter barriers than in SPS were needed for the RF operations necessary to collect the ions in a bucket. To not loose more than $80 \%$ of the ions a bucket of the size $14.4^{\circ}\left(7.2^{\circ}\right)$, corresponding to $4 \%(2 \%) \mathrm{DC}$, required barriers as thin as $300 \mathrm{~ns}(150 \mathrm{~ns})$. This is equivalent to $1 / 2(1 / 4)$ of the SPS cavities' filling time. A TW cavity with half the length would give half the filling time (according to $\tau=L_{T W} / v_{g}$ ) but it would also give half as much voltage. To compensate for that 4 times as much power for the cavity is needed since the shunt impedance, $R_{\text {shunt }}=\left|V_{\text {gap }}\right| /\left(2 P_{\text {loss }}^{2}\right)$, should be kept constant. For $\tau / 4$ the cavity would need to be fed with 16 times more power. Assuming TW cavities to achieve this would be too optimistic. We will here anyhow present the results from Barrier Bucket simulations (described in [9]) with bucket sizes DC $=4 \%$ (2\%) and barrier sizes $\tau=300 \mathrm{~ns}(150 \mathrm{~ns})$ and present the resulting bucket ion intensities. Based on the corresponding neutrino fluxes and suppression factors $(\mathrm{SF}=4 \%(2 \%))$ we will show the sensitivities.

\begin{tabular}{lcccc}
\hline & \multicolumn{2}{c}{${ }^{8} \mathbf{B}$} & \multicolumn{2}{c}{${ }^{8} \mathbf{L i}$} \\
\hline$N_{\text {SPS }}$ & \multicolumn{2}{c}{$1.69 \cdot 10^{13}$} & \multicolumn{2}{c}{$4.30 \cdot 10^{13}$} \\
\hline & SF 2\% & SF 4\% & SF 2\% & SF 4\% \\
\hline$R_{b}$ & 0.21 & 0.19 & 0.23 & 0.25 \\
$N_{\nu / \text { year }}$ & $3.49 \cdot 10^{18}$ & $3.25 \cdot 10^{18}$ & $7.57 \cdot 10^{18}$ & $7.99 \cdot 10^{18}$ \\
\hline
\end{tabular}

Table 1: $N_{\mathrm{SPS}}$, number of ions injected into the DR at each fill from the SPS, assuming no SPS charge intensity limitation. $R_{b}$, ratio between number of ions that decay inside the bucket over number of ions that escape the bucket before they decay, is given by Barrier Bucket simulations. $N_{\nu / \text { year }}$, annual (anti) neutrino rate from ${ }^{8} \mathrm{Li}\left({ }^{8} \mathrm{~B}\right)$ ions decaying in the bucket while it is in the useful straight fraction of the DR, is given by $N_{\nu / \text { year }}=N_{\mathrm{SPS}} R_{b} F_{s} \frac{t_{\text {year }}}{t_{\mathrm{SPS}}}$.

\subsection{Annual Neutrino Flux}

Neutrinos originating from ion decays inside the bucket are the useful neutrinos since they will arrive to the detector at those short fraction of times when it is "open" for data taking from the neutrino beam. The fraction of the DR that is filled with ions and the opened fraction of time of the detector are synchronized so that DC=SF. If the detector would not need any SF for the atmospheric background the DR could be fully filled with ions, $\mathrm{SF}=\mathrm{DC}=1$. Then the number of ions in the DR could accumulate to about $5 \cdot 10^{14}$ $\left(1 \cdot 10^{15}\right){ }^{8} \mathrm{~B}\left({ }^{8} \mathrm{Li}\right)$ ions. However due to ions leaving the bucket before decaying the accumulation in the bucket stops at about $35 \cdot 10^{12}$ number ${ }^{8} \mathrm{~B}$ ions and about $80 \cdot 10^{12}$ number ${ }^{8} \mathrm{Li}$ ions. The aim is to optimize the bucket ratio, $R_{b}$, i.e. the ratio between the number of ions that decay inside the bucket over the number of ions that escape the bucket before they decay. The simulations (described in [9]) indicate that barriers with filling time as short as $\tau=150 \mathrm{~ns}$ (300 ns) is needed for 2\% (4\%) DC to give $R_{b}$ around 0.2 (see table 1). To take the whole Beta Beam complex into account a Mathematica program, described in [10], was used. The number of neutrinos emitted per year from the bucket while it is in the useful straight fraction of the DR, $F_{s}$, is $N_{\nu / \text { year }}=N_{\mathrm{SPS}} R_{b} F_{s} \frac{t_{\text {year }}}{t_{\mathrm{SPS}}} . N_{\mathrm{SPS}}$ is the number of ions injected into the DR at each fill from the SPS with the repetition time $t_{\mathrm{SPS}}$ and $t_{\text {year }}$ is the time of operation per year (put to $10^{7} \mathrm{~s}$ ). Results are shown in table 1. Charge limitations of SPS was not taken into account so resulting fluxes are very over estimated. It will be shown now that the sensitivities are anyway not sufficient.

\section{Sensitivities}

From [3] one can conclude that FP6 needs a SF $<1 \%$. For an estimation of the sensitivities for the (anti) neutrino annual rates achieved for $R_{b} \approx 0.2$, i.e. $\left(7 \cdot 6 \cdot 10^{18}\right) 3.3 \cdot 10^{18}$, a simulation of the atmospheric background of muon neutrinos was made. A directional cut on the atmospheric background was made in the beam direction with the angle $\sqrt{1 / E(G e V)}$. The remaining background events were multiplied by 4 different SF's to achieve the sensitivity plots of $\theta_{13}$ and $\delta_{c p}$ shown in fig. 1 . Sensitivity plots of $\operatorname{sign}\left(\Delta m_{32}^{2}\right)$ were also made but are not shown since they do not change the conclusions. We see that 
a SF as strong as $0.01 \%$ would give same results as if there were no atmospheric background at all. Relaxing the SF to $0.1 \%$ does not decrease the sensitivity considerably but with $1 \%$ SF we see some effect of the atmospheric background. For SF as big as $10 \%$ the sensitivity gets unacceptably low. From this we conclude that for those fluxes achieved when $R_{b} \approx 0.2$ SF between $0.1 \%$ and $1 \%$ is needed.

(a)

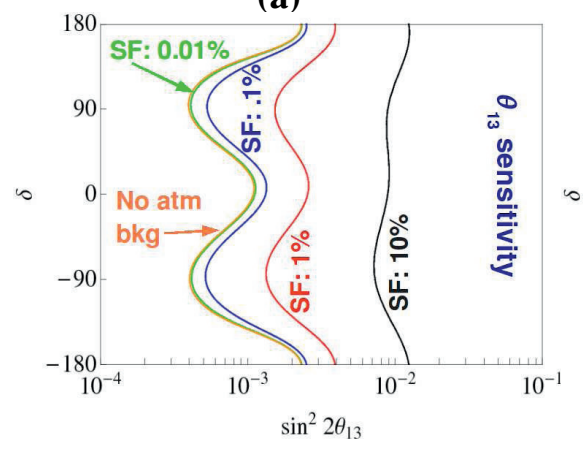

(b)

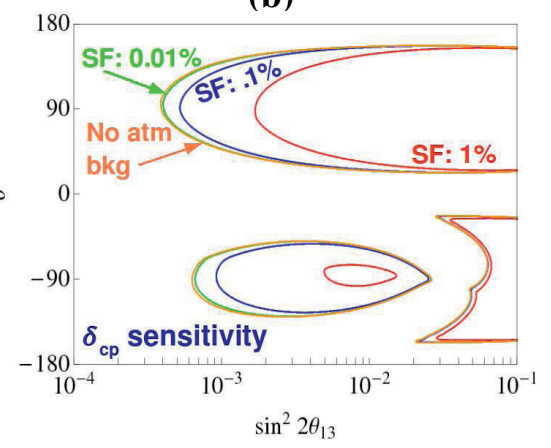

Fig. 1: The (a) $\theta_{13}$ and (b) $\delta_{c p}$ sensitivities for different suppression factors (SF).

\section{Conclusions}

We have seen, based on simulations of Barrier Bucket operations, that for a bucket with a size corresponding to $4 \%$ (2\%) DC of the DR barriers with filling times 1/2 (1/4) of the SPS cavities are necessary so that not more than $80 \%$ (i.e $R_{b} \approx 0.2$ ) of the ions escape the bucket before they decay. By additionally assuming an ion production rate of $10^{14}$ ions/s [5] for both ${ }^{8} \mathrm{~B}$ and ${ }^{8} \mathrm{Li}$ and no charge intensity limit in SPS a much too optimistic (anti) neutrino flux of $\left(7.57 \cdot 10^{18}\right) 3.25 \cdot 10^{18}$ were estimated. Even with these fluxes sensitivity plots of $\delta_{c p}$ and $\theta_{13}$ show that a suppression factor for the atmospheric background less than $1 \%$ would be needed. Since that suggests unrealistic RF cavities in the Decay Ring we conclude that the Barrier Bucket method is not optimal for the FP7 framework. We have however seen advantages with the Barrier Bucket method that would be of interest in the case of a more futuristic Beta Beam scenario with higher $\gamma$ since then the SF might not be as restricted as in the present case since the flux goes as $\gamma^{2}$. For the near future the prior RF scheme that proved to accomplish a duty cycle that followed the requirements, will have to be investigated also for the new ${ }^{8} \mathrm{~B}$ and ${ }^{8} \mathrm{Li}$ ions.

\section{Acknowledgments}

We are grateful for very useful discussions with and/or working material by M. Aiba, C. Bhat, M. Blaskiewicz, H. Damerau, R. Garoby, S. Hancock, E. Jensen, M. Mezzetto, S.Y. Lee, M. Lindroos and E. Shaposhnikova.

\section{References}

[1] EC http://cordis.europa.eu/fp6.

[2] A. Chance and S. Hancock, "Stacking simulations in the beta-beam decay ring,". EPAC06, Edinburgh, Scotland, 26-30 Jun 2006.

[3] M. Mezzetto Nucl. Phys. Proc. Suppl. 155 (2006) 214-217, hep-ex/0511005.

[4] EC http://cordis.europa.eu/fp7.

[5] C. Rubbia et al. Nucl. Instrum. Meth. A568 (2006) 475-487, hep-ph/0602032.

[6] T. Bohl, T. Linnecar, and E. Shaposhnikova EPAC 2000, Vienna, 26-30 Jun 2000.

[7] C. Hansen http://chansen.web.cern.ch/chansen/EURONU/dRAccumTW_Li_002.gif.

[8] C. Hansen http://chansen.web.cern.ch/chansen/EURONU/dRAccumTW_B_002.gif.

[9] C. Hansen et al., "Limitations in the use of barrier buckets in a beta beam decay ring," Proceedings of NUFACT09 (2009).

[10] E. Wildner et al. CERN-AB-2007-015. 